\title{
Simulation Of Ion Implantation Into Nuclear Materials And Comparison With Experiment
}

Article · June 2011

DOI: $10.1063 / 1.3586187$

CITATIONS

2

6 authors, including:

\section{Z. Insepov}

Nazarbayev University

146 PUBLICATIONS $\quad \mathbf{1 , 7 8 6}$ CITATIONS

SEE PROFILE

\section{Sergey V. Starikov}

Joint Institute for High Temperatures

58 PUBLICATIONS 320 CITATIONS

SEE PROFILE
READS

40

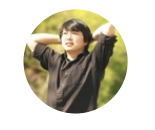

di Yun

Xi'an Jiaotong University

62 PUBLICATIONS 178 CITATIONS

SEE PROFILE

\section{Abdellatif Yacout}

Argonne National Laboratory

131 PUBLICATIONS 444 CITATIONS

SEE PROFILE

Some of the authors of this publication are also working on these related projects: 


\title{
Simulation of Ion Implantation into Nuclear Materials and Comparison with Experiment
}

\author{
Z. Insepov, ${ }^{\text {a }}$ A. Kuksin, ${ }^{\text {a }}$ J. Rest, ${ }^{\text {a }}$ S. Starikov, ${ }^{\text {a, }}$ A A. M. Yacout, ${ }^{\text {a }}$ A. Yanilkin, ${ }^{\text {a, }}$ * B. Ye,,${ }^{\text {a b D. }}$ \\ Yun ${ }^{\mathrm{a}, \mathrm{b}}$

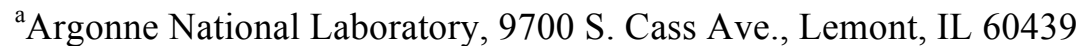 \\ ${ }^{b}$ University of Illinois at Urbana-Champaign, 104 S. Wright Street, Urbana, IL 61801
}

\begin{abstract}
Radiation defects generated in Mo formed by sub-MeV Xe ion implantations were studied by atomistic molecular dynamics based on interatomic potential matched to density functional calculations. The results of the simulations were qualitatively compared with defect distributions in $\mathrm{CeO}_{2}$ and $\mathrm{CeLaO}_{2}$ crystals used as surrogate materials for $\mathrm{UO}_{2}$ obtained from experiments by implantation of these ions at a dose of $1 \times 10^{17} \mathrm{ions} / \mathrm{cm}^{2}$ at several temperatures. A combination of in situ Transmission Electron Microscopy (TEM) and ex situ TEM experiments was used to study the evolution of defect clusters during implantation of $\mathrm{Xe}$ and $\mathrm{Kr}$ ions at energies of $150-700 \mathrm{keV}$, depending on the experimental conditions. The simulation and irradiation were performed on thin-film, single-crystal materials. The formation of defects, dislocation loops, and precipitates was studied by simulation and compared to experiment. Various sets of quantitative experimental results were obtained to characterize the dose and temperature effects of irradiation. These experimental results include size distributions of dislocation loops, voids, and gas bubble structures created by irradiation.
\end{abstract}

Keywords: Radiation defects, $\mathrm{Mo}, \mathrm{CeO}_{2}$, TEM, $\mathrm{Xe}$ and $\mathrm{Kr}$ ions, $\mathrm{MD}$ and $\mathrm{MC}$ simulations.

PACS: 61.72.uf, 61.72.uj, 61.72.up, 61.72.J-, 61.43.Bn, 61.43.Bn

\footnotetext{
* Permanent address: Joint Institute for High Temperatures RAS, 13 Izhorskaya , Moscow, 125412
} 


\section{INTRODUCTION}

Recently, a new application of ion implantation has been initiated that explores a unique capability in studying radiation effects and explore kinetics of defects in nuclear reactor environments [1,2]. This is a new and important research field because sustainable nuclear energy production must include a comprehensive analysis of nuclear fuel behavior. Fuel behavior codes are sensitive to materials parameters, many of which have large uncertainties or have not been measured and, thus, a complete understanding of radiation damage and swelling of nuclear fuels throughout the operating burnup and temperature regime is required [3].

Atomistic simulation is a powerful method for tracking defect accumulation during ion implantation and for estimating the values of critical materials properties and parameters used in kinetic fuel-behavior models. Whereas first-principles simulations are limited to a few hundred atoms at most, classical molecular dynamic (MD) calculations with many millions of atoms are routinely performed. However, the reliability and predictive power of classical MD depend crucially on the quality of the interatomic potential employed. For elementary solids, such potentials are usually obtained by adjusting a few potential parameters to optimally reproduce a set of reference data, which typically includes a number of experimental (or ab initio calculated) values such as lattice constants, cohesive energies, or elastic constants. However, in the case of more complex systems with a large variety of local environments and many potential parameters to be determined, such an approach cannot help. Here a new method of deriving realistic interatomic potentials is involved.

\section{SIMULATION}

The force matching method (FMM) provides a way to construct physically justified potentials even under such circumstances as absence of experimental data. This method provides new interatomic potentials that are obtained on the basis of synthesis of quantum and statistical 
mechanics. The idea is to compute forces and energies from first principles for a suitable selection of small reference systems and to adjust the parameters of the interatomic potential to optimally reproduce them $[4,5]$. The method allows creating correct potentials for simulation of various processes such as phase transitions, deformation at various temperatures, and stresses.

Using FMM, we developed an interatomic potential for Mo-Xe systems. The reference data was calculated by the VASP code [6]. The following parameters were used: the electron orbitals were represented by using plane waves, with a cut-off energy of $400 \mathrm{eV}$; the generalized gradient approximation for pseudopotential; 2x2x2-mesh in k-space. We used 81 various configurations with a total of 10,746 atoms. These configurations represent different Mo-Xe systems: 39 states with pure Mo (liquid and bcc solid states at different densities; solid states with self-interstitial atoms and/or vacancies and/or surface), 20 states with pure Xe (liquid and solid states), and 22 states with Mo-Xe (including a single Xe atom in pure Mo). Generally the matching was carried out with three value types: energy (only one in every configuration), stress tensor (six in every configuration), and forces (three components for each atom in every configuration). In the configurations with self-interstitial atoms (SIAs) and vacancies only the energy was taken into account.

The potential is represented in the form of the embedding atom method (EAM) with seven independent functions. This form is widely used for description of many-body interactions in metals and alloys. In our investigation the potential functions were set by splines with 10 independent parameters each. The FMM code PotFit [5] was used to search the optimal parameters of the potential.

The derived potential reproduces the reference data with high precision and provides a description of several thermophysical properties, which are not involved in the fitting procedure, in agreement with the experimental data. Table 1 shows a comparison of the basic Mo 
parameters calculated by using the new many-body potential with experiments. The Mo-Mo EAM potential presented reproduces the cold curve in agreement with the experimental data up to approximately $600 \mathrm{GPa}$ (corresponding compression $\mathrm{V} / \mathrm{V}_{0} \sim 0.5$ ). In addition, the description of thermal expansion is replicated well up to the melting point.

Table 2 shows a comparison of the defect properties calculated with the new EAM potential with the VASP data. Note that a difference between formation energies of defects is more important than the value of the energy itself. The most stable configurations of interstitial defects in Mo are $<111>$ dumbbell and $<111>$ crowdion with very small differences in formation energies. These configurations provide for one-dimensional migration of self-interstitial atoms at very low temperatures [7] in agreement with the resistivity recovery measurements following electron irradiation [8]. With increasing temperature the $<111>-<110>$ dumbbell transitions are activated, providing a rotation of the axis of migrating crowdions and hence providing a basis for a viable mechanism for three-dimensional diffusion.

Figure 1 compares the sputtering yields for $\mathrm{Xe}+$ ion bombardment of a Mo (100) surface calculated in the present paper with the experimental data obtained from the literature [9-14]. In addition, we have constructed two pair potentials for description of the Mo-Xe interaction. The parameters for the pair potential function for set \#1 yield data close to experiment at higher energies, namely, $E \sim 100 \mathrm{eV}$. However, the calculated yields are much higher than the data at energies lower than $60 \mathrm{eV}$. Set \#2 gives calculated yields close to experiment for both high- and low-energy regions. A new EAM interatomic potential for the Mo-Xe system gave sputtering yields close to the experimental data at the high-energy range (see Fig. 1). In what follows, set \#2 based on pair potential and the new EAM Mo-Xe potentials were applied for studying Xe bubble properties in Mo. 
Parallel MD calculations were carried out on a Blue Gene supercomputer by using a LAMMPS classical MD simulation package [15].

Figure 2 shows the time evolution of vacancies and self-interstitial defects in pure Mo irradiated with a $\mathrm{Xe}+$ ion, with energy of about $40 \mathrm{keV}$. The volume of the basic simulation cell was chosen to be $20 \times 20 \times 60 \mathrm{~nm}^{3}$. Several stages can be distinguished during the evolution of 40 keV cascade in Mo (Fig. 2): (a) penetration of Xe into the bulk, generation of numerous subcascades along the path, where the structure of the core of the cascade is close to amorphous with decreased density (up to $0.5 \mathrm{ps)}$; (b) fast motion of the leading atoms in the subcascades, where annealing of the amorphous core leads to the formation of vacancies in the center and interstitials in the vicinity (up to 2-4 ps); (c) fast motion of the leading atoms in the subcascades (their behavior is close to channeling), where the stopping result in the formation of interstitials quite far from the initial track (up to approx. $10 \mathrm{ps}$ ); and (d) nearly 1D migration of interstitials and clustering thereof, where recombination with vacancies is observed as well $(\sim 1-10 \mathrm{~ns}$ depending on the initial concentration). One should note that both sessile and mobile (also migrating one-dimensionally) SIA clusters are formed; their ratio depends on the temperature of the material.

The diffusivity of vacancies, SIAs, and mobile clusters from them was studied. Figure 3 shows the estimates of the self-diffusion coefficient in pure Mo at thermal equilibrium. The contributions from single vacancies and interstitials are taken into account: $D_{\text {self }}=c_{v} D_{v}+c_{i} D_{i}$. The temperature dependence of diffusivity of vacancies $D_{v}$ and interstitials $D_{i}$ is evaluated directly from MD simulations with the new EAM potential. Temperature-dependent equilibrium concentrations of the defects in Mo are estimated based on DFT calculations of the defects' formation enthalpy [16]. The self-diffusion coefficient calculated (Fig. 3) is in good agreement with the experimental data available for pure Mo $[17,18]$. 
Figure 4 shows the collision cascade where the ion track area is built up with vacancies and the self-interstitial clusters are formed in the outer regions. The MD simulation shows a single crystal of Mo after irradiation with a $50 \mathrm{keV} \mathrm{Xe} \mathrm{ion} \mathrm{at} \mathrm{a} \mathrm{dose} \mathrm{of} 1 \times 10^{12}$ ions $/ \mathrm{cm}^{2}$ at $300^{\circ} \mathrm{C}$. Clusters of interstitial atoms are formed by association of SIAs within 3 nanoseconds after the cascade initiation.

\section{EXPERIMENTAL}

Ion irradiation was done in the IVEM-Tandem facility at Argonne National Laboratory, and the scanning transmission electron microscopy study was carried out on JEOL 2200Fs TEM at the University of Illinois. Specifically, $500 \mathrm{keV} \mathrm{Xe} \mathrm{ions} \mathrm{were} \mathrm{implanted} \mathrm{into} \mathrm{a} \mathrm{single-crystal}$ $\mathrm{CeO}_{2}$ TEM specimen at $600{ }^{\circ} \mathrm{C}$ to an accumulated dose of $2 \times 10^{16}$ ions $/ \mathrm{cm}^{2}$. Figure 5 shows the atomic-level crystal structures of the specimen before and after irradiation. The specimen exhibits a perfect crystal structure before irradiation, Fig. 5(a), and remains crystalline after bombardment, Fig. 5(b). The areas with darker contrast in Fig. 5(b) suggest formation of defect clusters, ranging from $1 \mathrm{~nm}$ to $3 \mathrm{~nm}$ in diameter. However, the nature of these defect clusters is not clear yet. Therefore, any direct comparison of these TEM experiments to MD simulations is not yet possible because these two methods provide information on very different time scales.

Since the irradiation dose in the simulation was much lower $\left(1 \times 10^{12}\right.$ ions $\left./ \mathrm{cm}^{2}\right)$, the sizes of the clusters are smaller. Detailed analysis shows that they can be formed by two or three interstitial dislocation loops with diameters of 10-15 $\AA$.

Figures 6 and 7 show Xe gas bubble size distributions obtained at different ion irradiation dose levels on a $5 \%$ La-doped $\mathrm{CeO}_{2}$ single-crystal thin film and a $25 \%$ La-doped $\mathrm{CeO}_{2}$ single-crystal thin film, respectively. The thin films were grown on a $\mathrm{SrTiO}_{3}$ substrate with the molecular beam epitaxial technique. The Xe ion implantations were carried out with $700 \mathrm{keV}$ ex situ irradiations and $500 \mathrm{keV}$ in situ irradiations at $600{ }^{\circ} \mathrm{C}$. The sizes of the $\mathrm{Xe}$ gas bubbles were measured at 
implantation depths consistently around $80-100 \mathrm{~nm}$ on planar view, La-doped $\mathrm{CeO}_{2}$ single-crystal thin-film specimens after Xe ion implantations. The effective diameters of gas bubbles were obtained by measuring the area of the gas bubble features with the image-processing software ImageJ. Five sets of $32.5 \mathrm{~nm}$ by $32.5 \mathrm{~nm}$ boxes were drawn, and the densities of the Xe gas bubbles were measured with bubble feature profiling by ImageJ as well. The error bars in Figures 6 and 7 represent statistical errors within the five independent measurements.

These experimental bubble size distribution results are crucial to benchmark computer simulation results such as those from kinetic theory models or kinetic Monte Carlo models based on rates of elemental processes from MD as proposed in this study.

\section{SUMMARY}

Kinetic mesoscale models, such as those developed at Argonne National Laboratory are directly comparable to reactor experiments. Our new concept is based on kinetic rate-equations for radiation damage, energetics and kinetics of defects, and swelling of fuels as a function of temperature and burnup. Quantum and classical atomistic simulation methods are applied to increase our understanding of radiation damage, defect formation, and growth processes and to calculate the probabilities of elemental processes and reactions applicable to irradiated nuclear materials. Since the interaction potentials are critical for the new concept, they were developed based on a force-matching method data from ab initio calculations or were fitted to existing experimental data.

In the present paper, a new EAM potential is proposed for Mo-Xe systems that is constructed by the force matching method applied to a dataset of energies and forces from ab initio simulations with various structures. It is verified against existing surface erosion experimental data, basic thermophysical properties and ab initio data on energies of the defects. Several new 
Xe-Mo pair potentials were also parameterized by comparison of the calculated sputtering yield of a Mo-surface bombarded with Xe ions with experimental data.

\section{Acknowledgments}

This work was supported by the U.S. Dept. of Energy Office of Advanced Scientific Computing Research, Office of Science, under Contract DE-AC02-06CH11357. 


\section{REFERENCES:}

1. G.S. Was, J.T. Busby, T. Allen, E.A. Kenik, A. Jenssen, S.M. Bruemmer, J. Gan, A.D. Edwards, P.M. Scott, P.L. Andresen, J. Nucl. Mater. 300 (2002) 198.

2. Nuclear Fuel Safety Criteria. Technical Review, NEA/CSNI/R 25 (1999) 45.

3. J. Spino, D. Papaioannou, J. Nucl. Mater. 281 (2000) 146-162.

4. F. Ercolessi and J. B. Adams, Europhys. Lett. 26(8) (1994) 583.

5. P. Brommer and F. Gahler, Modelling Simulation Mater. Sci. Eng. 15 (2007) 295.

6. G. Kresse and J. Furthmuller, Phys. Rev. B 54 (1996) 11169.

7. S.P. Fitzgerald, D. Nguyen-Manh, Phys. Rev. Lett. 101 (2008) 115504.

8. P. Ehrhart, P. Jung, H. Shultz, H. Ullmaier, Atomic Defects in Metals, ed. by H. Ullmaier Landolt-Bornstein New Series, Group III, Vol. 25, Springer-Verlag, Berlin, 1991.

9. C.H. Weijsenfeld, A. Hoogendoorn, M. Koedam, Physica 27 (1961) 763.

10. D. Rosenberg, G.K. Wehner, J. Appl. Phys. 33 (1962) 1842.

11. J.J. Blandino, D.G. Goodwin, C.E. Garner, Diamond and Rel. Mat. 9 (2000) 1992-2001.

12. R.P. Doerner, D.G. Whyte, D.M. Goebel, J. Appl. Phys. 93 (2003) 5816.

13. R. Kolasinski, AIAA Paper No. 2005-3526, 2005.

14. E. Oyarzabal, J. H. Yu, R.P. Doerner, G.R. Tynan, K. Schmid, J. Appl. Phys. 100 (2006) 063301.

15. S. J. Plimpton, J. Comp. Phys. 117 (1995) 1.

16. T.R. Mattsson, N. Sandberg, R. Armiento, A.E. Mattsson, Phys. Rev. B 80 (2009) 224104.

17. K. Maier, H. Mehrer, and G. Rein, Z. fuer Metallkunde 70 (1979) 271.

18. G.B. Fedorov, E.A. Smirnov, V.N. Gusev, F.I. Zhomov and V.L. Gorbenko, in Metallurgy and Materials Science of Pure Metals, Atomizdat, Moscow, vol. 10, 1973, pp. 62-68. 
The submitted manuscript has been created by UChicago Argonne, LLC, Operator of Argonne National Laboratory ("Argonne"). Argonne, a U.S. Department of Energy Office of Science laboratory, is operated under Contract No. DE-AC02-06CH11357. The U.S. Government retains for itself, and others acting on its behalf, a paid-up nonexclusive, irrevocable worldwide license in said article to reproduce, prepare derivative works, distribute copies to the public, and perform publicly and display publicly, by or on behalf of the Government. 
Table 1: Comparison of simulation results (with new EAM potential) with experimental data for pure Mo

\begin{tabular}{|l|l|l|l|l|l|}
\hline & $\begin{array}{l}\text { Cohesive } \\
\text { energy }(\mathrm{eV})\end{array}$ & $\begin{array}{l}\text { Lattice } \\
\text { parameters }(\AA)\end{array}$ & C11 $(\mathrm{GPa})$ & C12 $(\mathrm{GPa})$ & $\begin{array}{l}\text { Melting } \\
\text { temperature }(\mathrm{K})\end{array}$ \\
\hline Simulation & 6.91 & 3.1469 & 560 & 225 & 2630 \\
\hline Experiment & 6.82 & 3.147 & 460 & 176 & 2890 \\
\hline
\end{tabular}


Table 2: Comparison of simulation results (with new EAM potential) with VASP calculation for Mo defects

\begin{tabular}{|l|c|c|c|c|}
\hline & $\begin{array}{l}\text { Formation energy of } \\
\text { Crowdion }<111>(\mathrm{eV})\end{array}$ & $\begin{array}{l}\text { Formation energy of } \\
\text { Dumbbell }<111> \\
(\mathrm{eV})\end{array}$ & $\begin{array}{l}\text { Formation energy of } \\
\text { Dumbbell }<110>(\mathrm{eV})\end{array}$ & $\begin{array}{l}\text { Formation energy } \\
\text { of vacancy }\end{array}$ \\
\hline Simulation & 6.42 & 6.43 & 6.67 & 2.79 \\
\hline VASP & 6.89 & 6.88 & 7.02 & 2.40 \\
\hline
\end{tabular}


Figure captions:

Figure 1. Comparison of the sputtering yield of a Mo (100) surface bombarded by accelerated $\mathrm{Xe}+$-ions interacting with Mo atoms via a Morse potential with the experimental data from Refs. [33-38] (see also Fig. 2).

Figure 2. Time evolution of defect distribution after $40 \mathrm{keV}$ cascade in pure Mo. The volume of the basic simulation cell is $20 \times 20 \times 60 \mathrm{~nm}^{3}$.

Figure 3. Self-diffusion coefficient in pure Mo at thermal equilibrium: comparison of MD calculations with the new EAM potential with two existing experimental data sets [16, 17].

Figure 4. MD simulation of radiation track formation in single-crystal Mo after irradiation with a $50 \mathrm{keV} \mathrm{Xe}$ ion at a dose of $1 \times 10^{12}$ ions $/ \mathrm{cm}^{2}$ at $300{ }^{\circ} \mathrm{C}$. Clusters of interstitial atoms are formed by association of SIA within 3 nanoseconds of simulation.

Figure 5. Scanning TEM micrograph of single-crystal $\mathrm{CeO}_{2}$ (a) before irradiation and (b) after irradiated with $500 \mathrm{keV} \mathrm{Xe}$ to a dose of $2 \times 10^{16} \mathrm{ions} / \mathrm{cm}^{2}$ at $600{ }^{\circ} \mathrm{C}$. Electron beam direction is along $<001>$.

Figure 6. Bubble size distribution from $700 \mathrm{keV}$ and $500 \mathrm{keV} \mathrm{Xe} \mathrm{irradiations} \mathrm{at} 600{ }^{\circ} \mathrm{C}$ on $5 \%$ La-doped $\mathrm{CeO}_{2}$ (lines are drawn between data points only to guide the eyes).

Figure 7. Comparison of bubble size distributions between different doses with $700 \mathrm{keV} \mathrm{Xe} \mathrm{ex}$ situ irradiations and $500 \mathrm{keV} \mathrm{Xe}$ in situ irradiations at $600{ }^{\circ} \mathrm{C}$ on $25 \% \mathrm{La}$-doped $\mathrm{CeO}_{2}$ (lines are drawn between data points only to guide the eyes). 


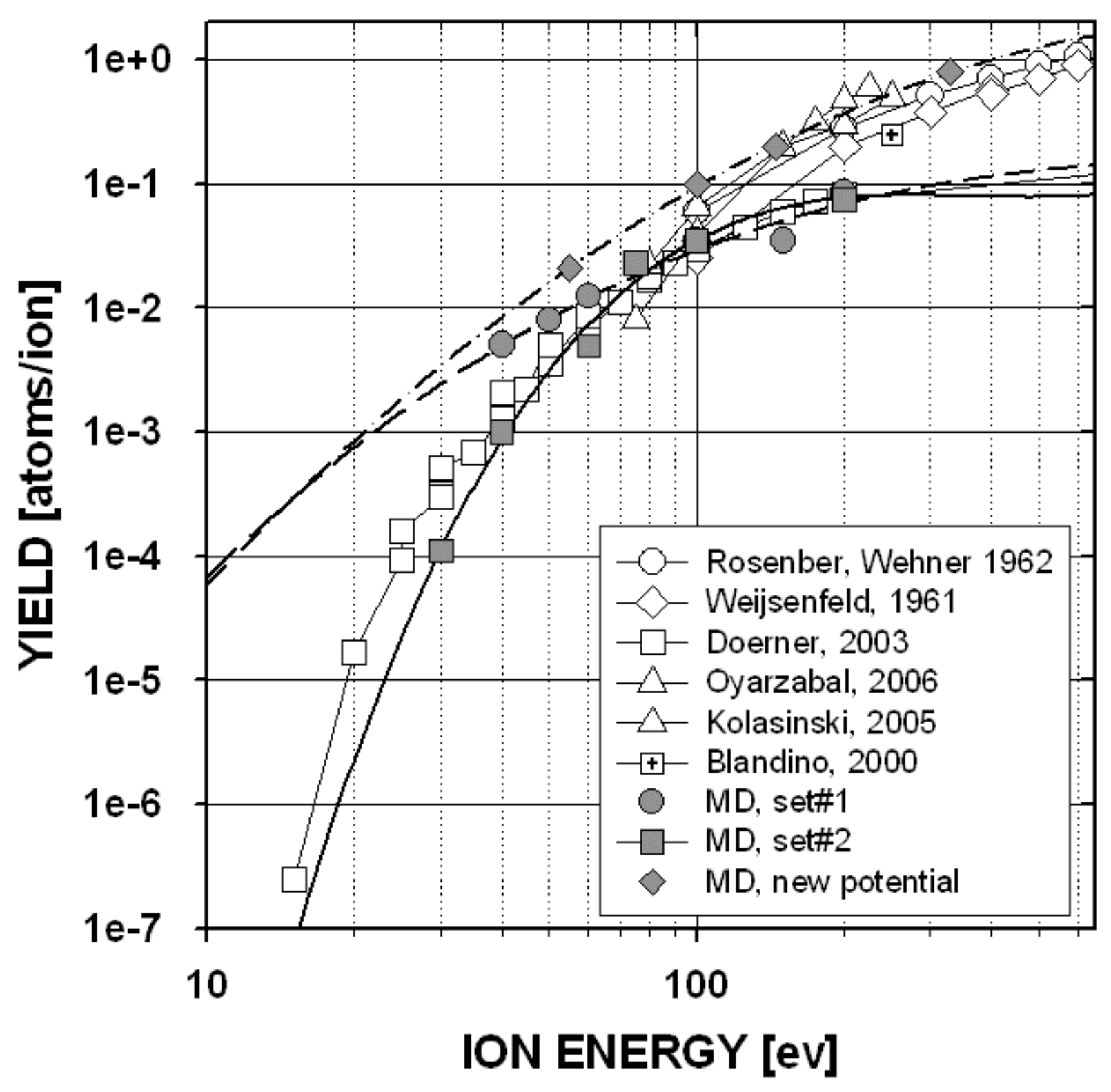

Figure 1 


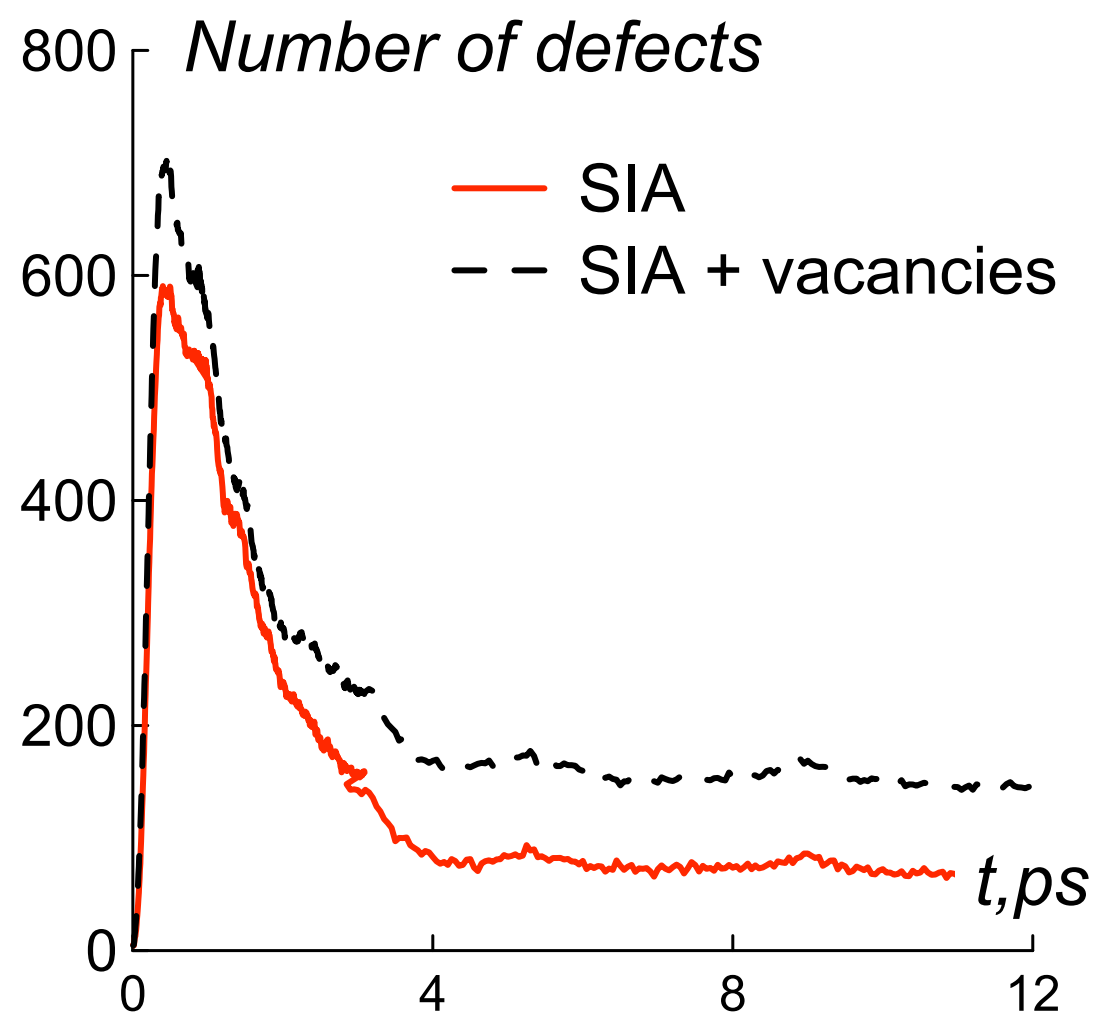

Figure 2 


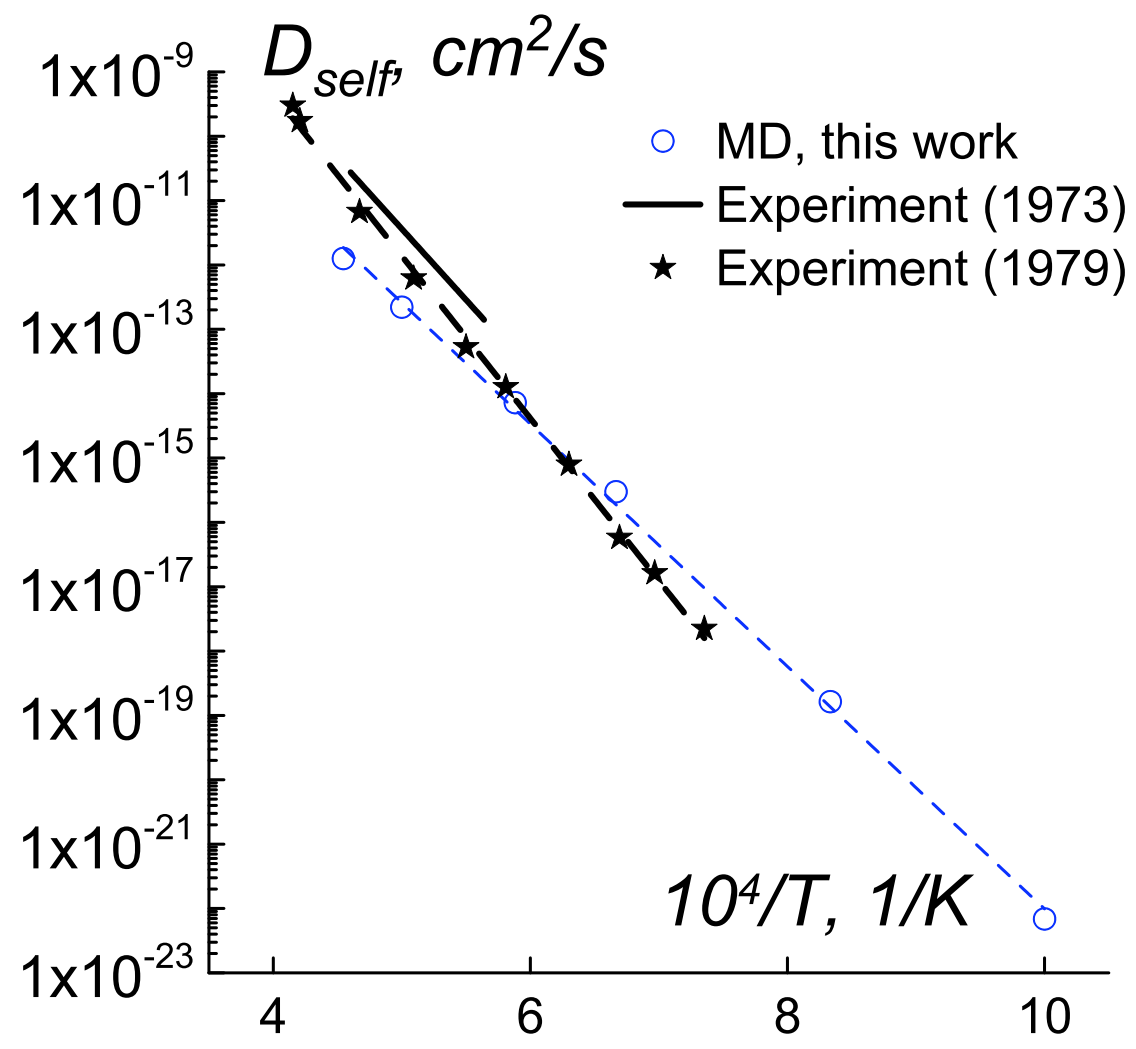

Figure 3 


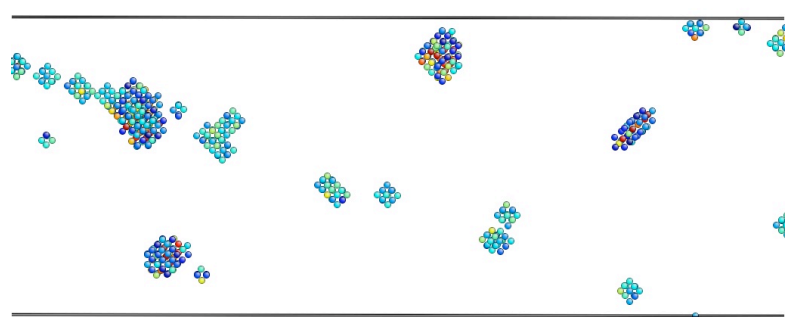

Figure 4 
IBMM 2010

Montréal, August 22-27, 2010
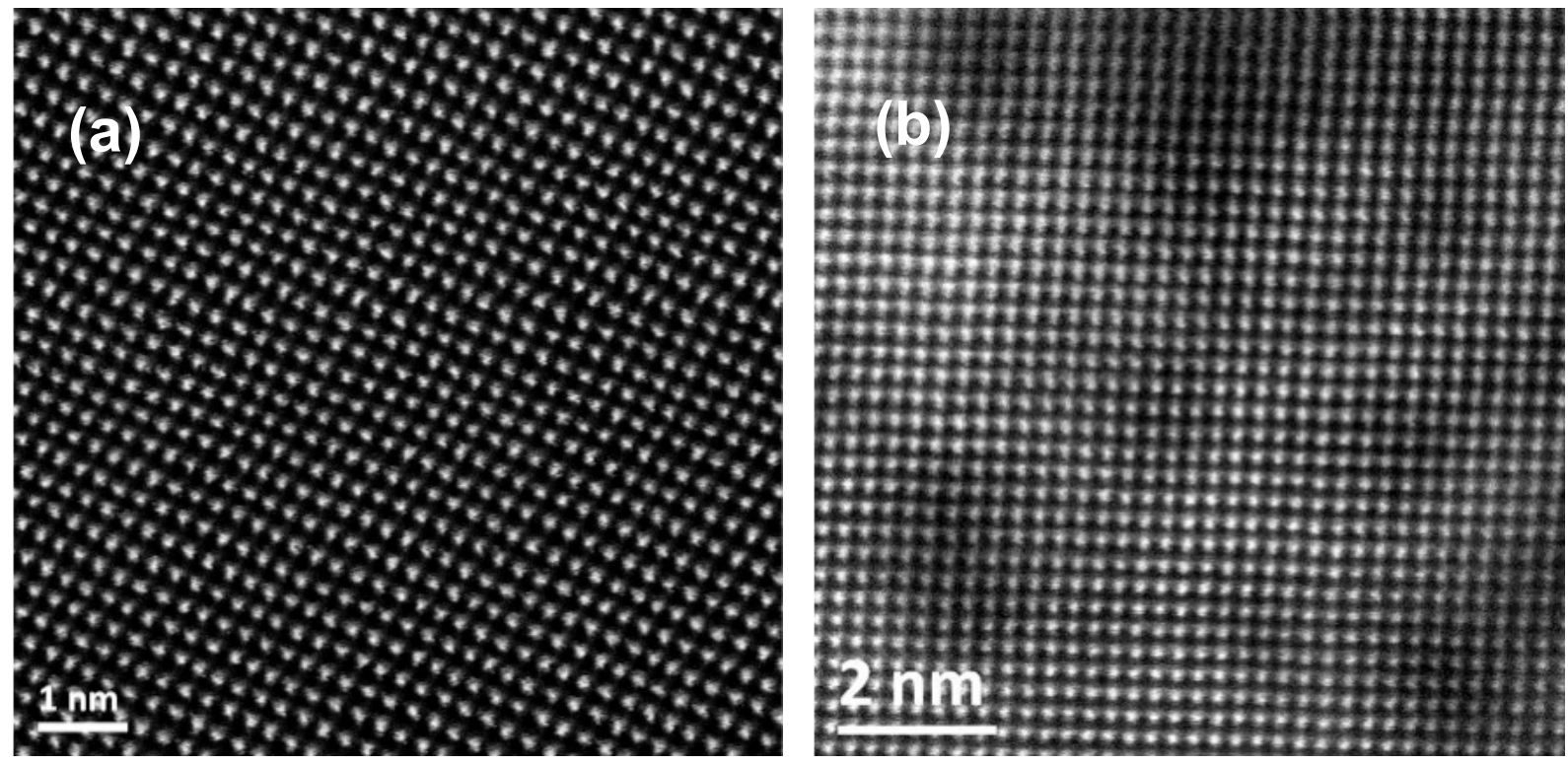

Figure 5 


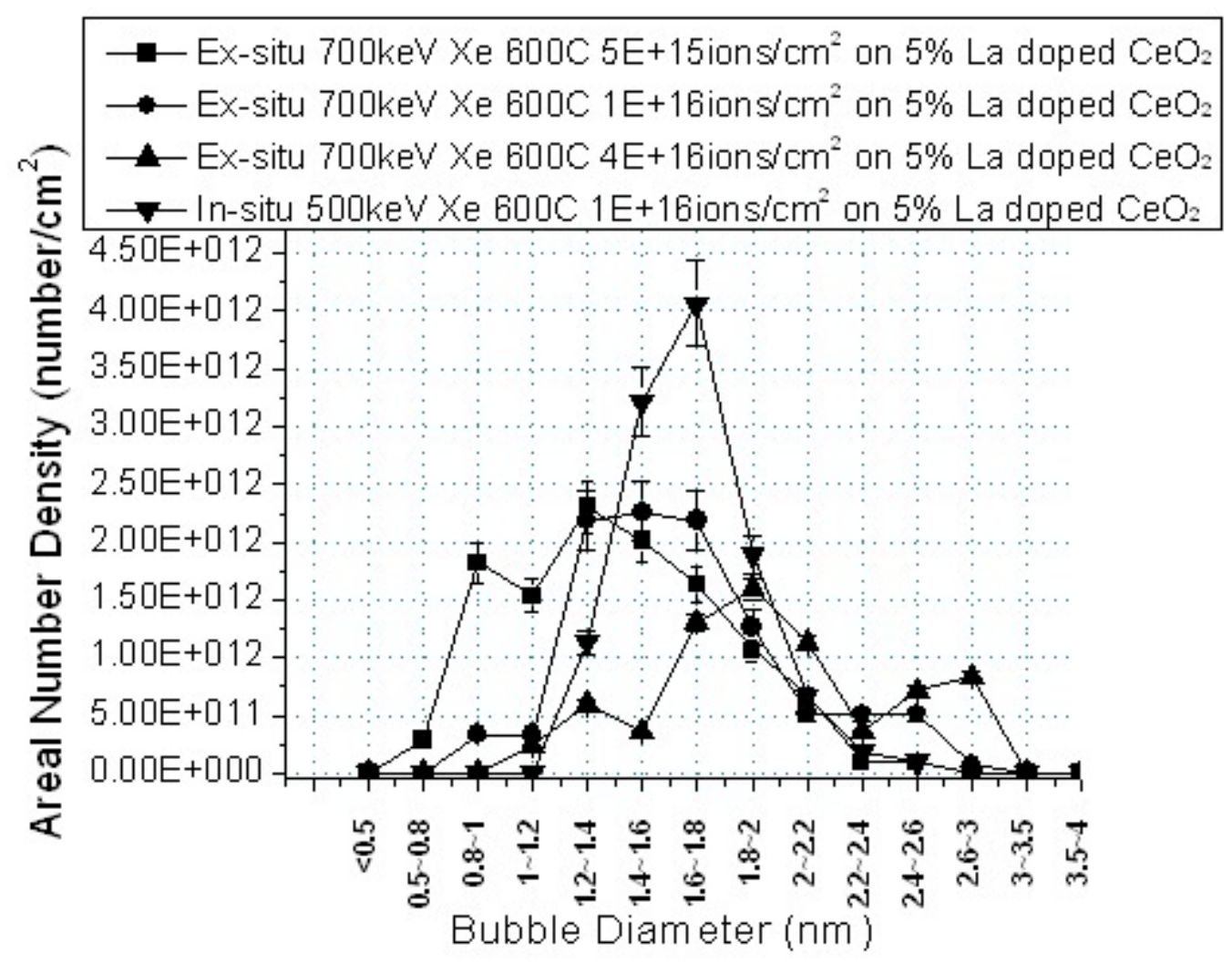

Figure 6 


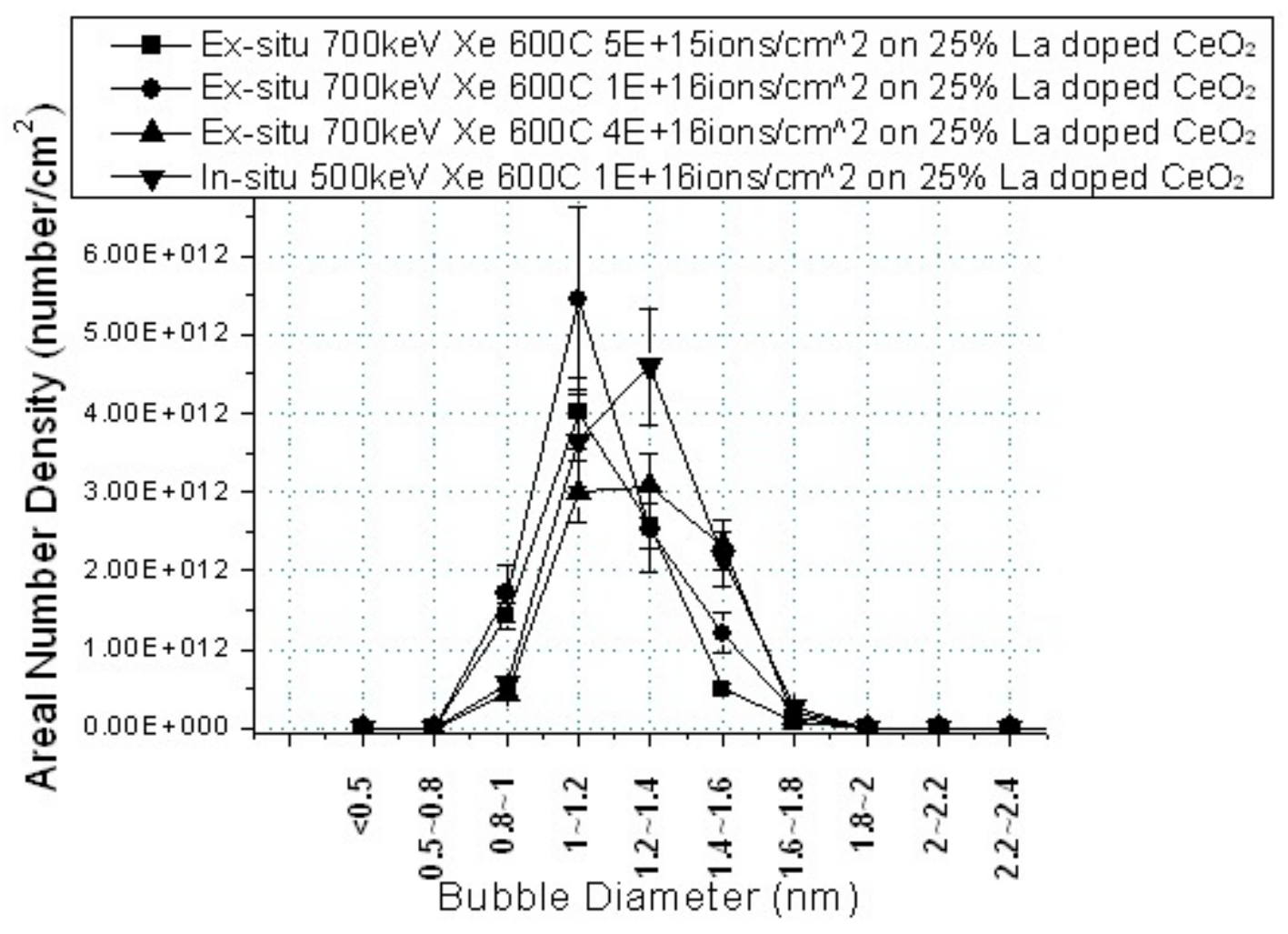

Figure 7 